\title{
Coagulation Abnormalities in Pregnant Women with COVID-19
}

\author{
Vladimir Y Khryshchanovich, $M D^{1^{*}}$ and Natalia Y Skobeleva, $B S^{2}$ \\ ${ }^{1}$ Professor, $2^{\text {nd }}$ Department of Surgical Diseases, Belarusian State Medical University, Minsk, Belarus \\ ${ }^{2}$ Assistant Professor, Department of Obstetrics and Gynecology, Belarusian State Medical University, \\ Minsk, Belarus
}

*Corresponding author: Vladimir Khryshchanovich, MD, Professor of the $2^{\text {nd }}$ Department of Surgical Diseases, Belarusian State Medical University, Minsk, Dzerzhinski Ave., 83., 220116, Republic of Belarus, Tel: +375-29624-55-78

Late pregnancy and the early postpartum period are characterized by the so-called physiological hypercoagulation: The concentration of blood clotting factors I, II, VIII, IX, X increases twice, the functional activity of platelets increases, and the fibrinolytic activity of serum decreases [1]. On the one hand, the prothrombotic status reduces the risk of massive blood loss in childbirth, and on the other - creates prerequisites for the development of venous thromboembolism [2]. In the context of assessing the perinatal risk of venous thromboembolism, pregnant women with coronavirus disease 2019 (COVID-19) deserve special attention [3]. As it became known, SARS-CoV-2 infection during pregnancy may be an additional trigger of severe thrombotic events [4]. However, to date, there are isolated reports in the available literature regarding the hemostasiological and pro-inflammatory status in pregnant women with COVID-19, while the effect of coronavirus infection on perinatal coagulation parameters remains unexplored [5].

The study included pregnant women with symptomatic COVID-19 and without SARS-CoV-2 infection who were admitted to the hospital in the period from December 1, 2020 to February 28, 2021, without obstetric complications, a history of confirmed thrombophilia and venous thromboembolism. SARSCoV-2 infection was confirmed by reverse transcription polymerase chain reaction (PCR) of a nasopharyngeal smear. Blood samples were collected on the day of admission for comparative analysis of hematological parameters (prothrombin and activated partial thromboplastin time [aPTT], fibrinogen, D-dimer, international normalized ratio [INR], C-reactive protein [CRP], platelets, white blood cells [WBC], erythrocyte sedimentation rate [ESR]) in both groups of pregnant women. 33 patients with COVID-19 and 30 women without SARS-CoV-2 infection aged 30.5 [27.7-34] and 25.5 [23.3-32.5] years, respectively, were selected for the study by sequential sampling. In all cases, the terms of pregnancy corresponded to the third trimester. The clinical manifestations of COVID-19 were characterized by mild respiratory symptoms (dry cough, slight shortness of breath), fever, and asthenia.

Significant inter-group differences in the analyzed laboratory parameters were found only in the concentrations of D-dimer and CRP (Table 1). The remaining parameters of coagulation, as well as the level of WBC and ESR, did not have statistically significant differences between the compared groups. At the same time, in pregnant women with COVID-19, in contrast to women without SARS-CoV-2 infection, there was a tendency to multidirectional hemostasiological deviations - lengthening of aPTT, an increase in INR and fibrinogen concentration, a decrease in platelet levels. There were no peripartum venous thromboembolic events in the compared groups according to Doppler ultrasonography data. Recent publications have indicated a direct correlation between the severity of SARS-CoV-2 infection and impaired coagulation parameters in patients with severe COVID-19 $[6,7]$. However, the inclusion of women with mild manifestations of COVID-19 in our study does not allow us to extend the results to the population of pregnant women with severe SARS-CoV-2 infection. 
Table 1: Coagulation and inflammation parameters of hospitalized women during pregnancy with or without COVID-19 infection.

\begin{tabular}{|l|l|l|l|}
\hline Parameters, Me $\left(\mathbf{Q}_{\mathbf{1}}-\mathbf{Q}_{\mathbf{3}}\right)$ & COVID-19 $(\mathbf{n}=\mathbf{3 3})$ & Control $(\mathbf{n}=\mathbf{3 0})$ & $P$ value \\
\hline aPTT, sec & $29.35[26.30-30.55]$ & $28.90[26.98-30.15]$ & 0.96 \\
\hline PT, sec & $10.75[10.30-11.95]$ & $10.75[10.40-11.18]$ & 0.45 \\
\hline INR & $0.93[0.89-0.99]$ & $0.89[0.84-0.94]$ & 0.06 \\
\hline D-dimer, $\mu$ g/ml & $0.96[0.89-1.37]$ & $0.39[0.33-0.43]$ & $<0.001$ \\
\hline Fibrinogen, g/l & $6.30[5.41-8.00]$ & $6.14[5.59-6.83]$ & 0.53 \\
\hline CRP, mg/l & $9.60[1.58-29.23]$ & $1.55[1.00-3.40]$ & 0.003 \\
\hline Platelets, $\times 10^{\prime / l}$ & $215.5[164.2-278.0]$ & $221.5[177.8-266.00]$ & 0.64 \\
\hline ESR, mm/h & $30.50[20.00-45.25]$ & $34.00[23.25-42.25]$ & 0.88 \\
\hline White blood cells, $\times 10^{9 / l}$ & $8.49[7.23-10.09]$ & $8.84[6.71-11.23]$ & 0.76 \\
\hline
\end{tabular}

aPTT: Activated Partial Thromboplastin Time; PT: Prothrombin Time; INR: International Normalized Ratio; CRP: C-Reactive Protein; ESR: Erythrocyte Sedimentation Rate

In conclusion, it should be noted that most of the studied hematological parameters were almost identical in both groups. At the same time, in patients with symptomatic COVID-19, the levels of D-dimer and CRP were about 2.5 and 6 times higher, respectively, compared to pregnant women without SARS-CoV-2 infection. Prevention of perinatal COVID-19-associated venous thromboembolism seems to require individual assessment of at least routine hemostasiogram parameters and inflammation in all pregnant women with a positive result of a PCR test for SARS-CoV-2 infection.

\section{Conflicts of Interests}

The authors report no conflict of interest.

\section{References}

1. O'Connor DJ, Scher LA, Gargiulo NJ III, Jang J, Suggs WD, et al. (2011) Incidence and characteristics of venous thromboembolic disease during pregnancy and the postnatal period: A contemporary series. Ann Vasc Surg 25: 9-14.
2. Galambosi PJ, Gissler M, Kaaja RJ, Ulander VM (2017) Incidence and risk factors of venous thromboembolism during postpartum period: A population-based cohortstudy. Acta Obstet Gynecol Scand 96: 852-861.

3. Bates SM, Rajasekhar A, Middeldorp S (2018) American Society of Hematology 2018 guidelines for management of venous thromboembolism: Venous thromboembolism in the context of pregnancy. Blood Adv 27: 3317-3359.

4. Martinelli I, Ferrazzi E, Ciavarella A, Erra R, lurlaro E, et al. (2020) Pulmonary embolism in a young pregnant woman with COVID-19. Thromb Res 191: 36-37.

5. Ciavarella A, Erra R, Abbattista M, lurlaro E, Anzoletti MB, et al. (2021) Hemostasis in pregnant women with COVID-19. Int J Gynaecol Obstet 152: 268-269.

6. Peyvandi F, Artoni A, Novembrino C, Aliberti S, Panigada $M$, et al. (2021) Hemostatic alterations in COVID-19. Haematologica 106: 1472-1475.

7. Thachil J, Tang N, Gando S, Falanga A, Cattaneo M, et al. (2020) ISTH interim guidance on recognition and management of coagulopathy in COVID-19. J Thromb Haemost 18: 1023-1026. 